UCRL-JC-124401

Preprint

\title{
Conceptual Design for a Neutron Imaging System for Thick Target Analysis Operating in the 10-15 MeV Energy Range
}

\author{
Frank Dietrich \\ James Hall \\ Clint Logan
}

This paper was prepared for submittal to

14th International Conference on the Application of Accelerators

in Research and Industry

Denton, TX

November 6-9, 1996

September 11, 1996

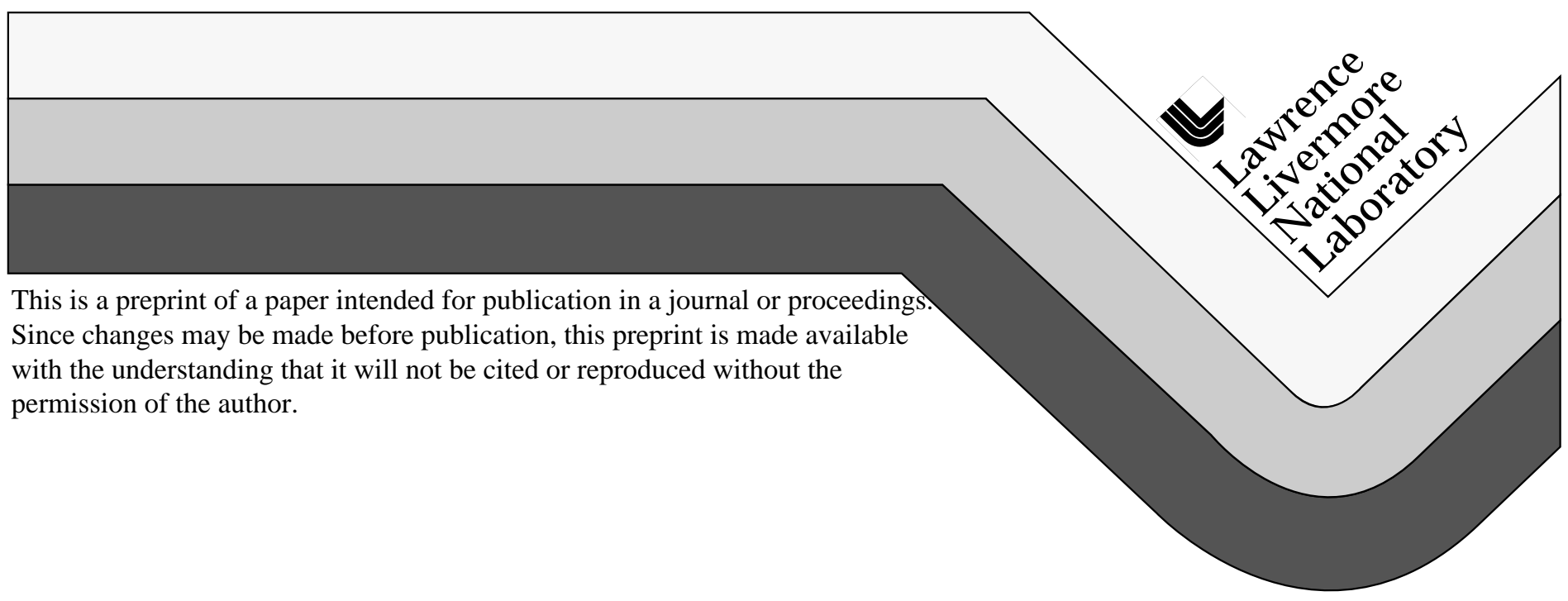




\section{DISCLAIMER}

This document was prepared as an account of work sponsored by an agency of the United States Government. Neither the United States Government nor the University of California nor any of their employees, makes any warranty, express or implied, or assumes any legal liability or responsibility for the accuracy, completeness, or usefulness of any information, apparatus, product, or process disclosed, or represents that its use would not infringe privately owned rights. Reference herein to any specific commercial product, process, or service by trade name, trademark, manufacturer, or otherwise, does not necessarily constitute or imply its endorsement, recommendation, or favoring by the United States Government or the University of California. The views and opinions of authors expressed herein do not necessarily state or reflect those of the United States Government or the University of California, and shall not be used for advertising or product endorsement purposes. 


\title{
Conceptual Design for a Neutron Imaging System for Thick Target Analysis \\ Operating in the $10-15 \mathrm{MeV}$ Energy Range
}

Frank Dietrich, James Hall and Clint Logan

Lawrence Livermore National Laboratory

P.O. Box 808, M/S L-028, Livermore, CA 94551-9900

\begin{abstract}
Fast neutron imaging offers the potential to be a powerful non-destructive inspection tool for evaluating the integrity of thick sealed targets. This is particularly true in cases where one is interested in detecting voids, cracks or other defects in low-Z materials (e.g. plastics, ceramics, salts, etc.) which are shielded by thick, high-Z parts. In this paper we present the conceptual design for a neutron imaging system for use in the $10-15 \mathrm{MeV}$ energy range and discuss potential applications in the area of nuclear stockpile stewardship. The background of this project, currently under development at LLNL, will be outlined and computer simulations will be presented which predict system performance. Efforts to assess technical risks involved in the development of the system will be discussed and the results of a recent experiment designed to evaluate background radiation levels will also be presented.
\end{abstract}

Keywords: nonintrusive inspection, neutron imaging, numerical modeling, nuclear stockpile stewardship

\section{INTRODUCTION}

Neutron imaging systems operating in the low energy range $(\leq 1 \mathrm{MeV})$ have been used in non-destructive testing for a number of years now; however, their use has generally been limited to the inspection of relatively small targets. We believe that fast neutron imaging at higher energies $(\geq 8 \mathrm{MeV})$ may offer the potential to be a powerful non-destructive inspection tool for use in evaluating the integrity of thick sealed targets. One possible application of particular interest to us at LLNL lies in the area of nuclear stockpile stewardship.

Changes in the US nuclear stockpile and the erosion of its associated production complex over the past decade have made the non-destructive (and non-intrusive) surveillance of weapons systems an increasingly important issue. Historically, when the integrity and/or reliability of a stockpiled weapons system has been called into question, the inspection procedures required to re-certify the system (which usually involve the partial or total disassembly of one or more devices) have proven to be expensive and generally irreversible. The development of advanced photon imaging techniques (e.g. gamma radiography and tomography) has greatly enhanced our stockpile surveillance capabilities; however, while photon imaging has proven to be an adequate tool for inspecting the condition of heavy metal (high-Z) parts within an object, it has not proven to be an effective technique for determining whether voids, cracks or other significant structural defects exist in the lighter (low-Z) materials (e.g. plastics, ceramics, salts, etc.) within the object, particularly in cases where the low- $Z$ materials are shielded by thick, high- $Z$ components. Since neutron interaction cross sections scale much more slowly with $Z$ than do photon cross sections, fast neutron imaging should be far more sensitive than photon imaging to defects such as these in low-Z materials.

We recently collaborated in experiments at the Los Alamos Nuclear Science Center (LANSCE) aimed at establishing a proof-of-principle for the radiography of thick targets using very high energy neutrons $(\approx 40-400 \mathrm{MeV})^{1}$. In those experiments (cf. Figure 1), an assembly consisting of a low-Z disk (LiD, $2.54 \mathrm{~cm}$ thickness) sandwiched between two high-Z slabs (uranium, $5.08 \mathrm{~cm}$ thickness) was used as a phantom target. Several small holes $(4-12 \mathrm{~mm}$ in diameter) drilled all or part way through the disk were used to simulate defects in the low-Z material. A positionsensitive multiwire detector with a tungsten converter plate was used to record an image of the transmitted neutrons with a spatial resolution of $\approx 1 \mathrm{~mm}$. The success of these early experiments has lead to the initiation of a major program to develop high energy $(\approx 40-400 \mathrm{MeV})$ neutron imaging techniques at LANL.

In view of the fact that the neutron source requirements for some imaging applications (including, but certainly not limited to, nuclear stockpile stewardship) may be more easily met at much lower energies than those used in the LANSCE experiments, we are currently studying the feasibility of doing neutron imaging in the $10-15 \mathrm{MeV}$ range. While the overall neutron transmission through thick targets is somewhat lower in this range than at higher energies (cf. Figure 2), this disadvantage is offset by the ability to use plastic-scintillator-based detectors which are more efficient than the converter-based detectors used for high-energy neutrons. In addition, the transmission contrast ratio in low-Z materials (i.e. the relative change in the transmitted intensity when a small amount of material is removed from the beam path) is also more favorable at lower neutron energies thereby making it easier to detect defects in 
those materials. We have recently developed the conceptual design for a neutron imaging system for use in the 10 $15 \mathrm{MeV}$ range that we believe will have reasonable detection efficiency $(\approx 30 \%)$ and spatial resolution $(\approx 1 \mathrm{~mm}$ at the target). The proposed system is described in $\S 3$ below.

\section{NUMERICAL SIMULATIONS}

We began our investigation of fast neutron imaging by modeling the high-energy LANSCE proof-of-principle experiments using 14-MeV neutrons with the LLNL-developed "COG" Monte Carlo radiation transport code. ${ }^{2}$ We have also used simple analytical models to simulate tomographic experiments and image reconstruction techniques on suitable objects. The results of the COG runs at $14 \mathrm{MeV}$ (cf. Figure 3) show that neutron imaging in the 10 - 15 $\mathrm{MeV}$ energy range should be capable of revealing structural detail in thick targets similar to that seen in the much higher-energy LANSCE experiments. These results, along with our tomographic simulations of other phantom targets, suggest that it should be possible to detect defects $\approx 1 \mathrm{~mm}$ in diameter in shielded, low-Z materials during an exposure of a few hours using $10-15 \mathrm{MeV}$ neutrons from a source of practical size (we estimate that an integrated flux at the object $\approx 10^{9} \mathrm{n} / \mathrm{cm}^{2}$ will be required to achieve this level of sensitivity).

Optimizing the design of a full-scale neutron imaging system capable of inspecting a fully weaponized nuclear device (including minimizing the required source strength and mitigating the effects of multiple neutron scattering) will require a careful evaluation of radiation background levels and shielding requirements. We are currently studying these and other engineering design issues and evaluating various tomographic reconstruction algorithms using a combination of detailed computer simulations and experiments at LLNL and other facilities.

\section{CONCEPTUAL DESIGN}

The fast neutron imaging system that we have proposed for use in nuclear stockpile stewardship applications will consist of three primary components (cf. Figure 4): a high-luminosity neutron source operating in the $10-15$ $\mathrm{MeV}$ energy range (nominal output $\approx 10^{12} / 4 \pi \mathrm{n} / \mathrm{s} / \mathrm{sr}$ in the forward direction with an effective spot size $\approx 1 \mathrm{~mm}$ in diameter), a low-mass rotation stage to support and manipulate the object under inspection (thus allowing for tomographic imaging) and the imaging package itself. Based on our numerical simulations of several proposed facility layouts, the object to be inspected will probably be placed midway between the source and the imaging plane (2:1 magnification) to minimize radiation backgrounds due to multiple neutron scattering within the object itself. If an isotropic DT neutron generator is used, then the shielding wall shown between the source and the object may be required to mitigate radiation backgrounds due to room scattering; however, if an accelerator-driven DD source is used, then the neutron beam will be naturally collimated in the forward direction $\left(0^{\circ}\right)$ due to beam kinematics and room scattering should not be a problem and the wall would probably not be required. The second shielding wall (between the object and the imaging package) will be used to protect potentially sensitive electronic components within the imaging package and is considered essential for background reduction.

The design of the imaging package itself is based on proven Nuclear Test Program technology (cf. Figure 5). We propose to use a simple plastic scintillator fluor (e.g. BC-408) as an imaging plane which would then be viewed by one or more standard CCD cameras. Our calculations indicate that a fluor with a thickness $\approx 4 \mathrm{~cm}$ should have a detection efficiency in excess of $30 \%$ for $14 \mathrm{MeV}$ neutrons (this compares to an efficiency of $\approx 0.3 \%$ for the conversion plate used in the higher-energy LANSCE experiments). The camera assembly will include a fast (f/1.2) lens coupled to a standard, back-thinned, high-resolution $(\approx 512 \times 512)$ CCD imaging chip operating at liquid nitrogen temperatures to minimize thermal electronic noise. A thin metallic turning mirror (e.g. NiCu) will be used to reflect light from the scintillator fluor into the camera system which will be located in a shielded enclosure well out of the neutron beam path. Our calculations indicate that the camera should be capable of sensing the light generated by a single neutron interacting within the fluor; thus an image intensifier should not be necessary unless we find that it is needed for some other reason (e.g. time gating). The spatial resolution of the imaging system should be $\approx 1.50 \mathrm{~mm}$ at the fluor (this would correspond to a resolution of $\approx 0.75 \mathrm{~mm}$ at the object location for the $8 \mathrm{~m}, 2: 1$ magnification facility layout currently under consideration). We plan to build a prototype of this imaging system during the early part of FY97 and test it against suitable phantom targets at the Ohio University Accelerator Laboratory (OUAL) in Athens, Ohio. OUAL features accelerator-driven DD and DT neutron sources and a shielded detector cave similar to that envisioned in an actual neutron imaging facility.

\section{TECHNICAL RISK ASSESSMENT}

We are currently working to improve our understanding of the technical risks associated with developing and using a fast neutron imaging system such as the one proposed here for nuclear stockpile surveillance. We recently completed an experiment designed to measure the scattered radiation background levels in a prototypical imaging system environment. The experiment was done using $10 \mathrm{MeV}$ neutrons from an accelerator-driven DD source at OUAL and utilized an NE-213 liquid-crystal scintillator detector to characterize radiation backgrounds in terms of 
energy, time-of-flight and particle type (i.e. neutrons vs. gammas). By comparing spectra taken with and without heavy iron shadow bars between the source and the detector, we were able to determine that radiation background levels due to scattering from sources external to the object under inspection (i.e. room scattering) should be much smaller than the direct (unscattered) beam transmitted through the object when a DD source is used. These results also indicate that accelerator pulsing should not be required to mitigate scattered backgrounds.

Several other technical risk issues which we have already addressed or are currently investigating involve the potentially adverse effects of prolonged exposure to fast neutrons on various materials typically found in a nuclear device, in particular uranium metal and lithium salts. In order to avoid making a device difficult to handle after the inspection process, it is essential that the radiation dose due to induced activation of the device assembly fall well below the intrinsic radiation level of the device itself within some reasonable time following the irradiation $(\approx 24$ hours). Detailed numerical simulations for one system currently in the stockpile suggest that activation should not be a problem for the materials in the weapon's aeroshell or component support structures; however, our codes cannot account for activation of the uranium itself. This will be addressed separately in experiments planned for early FY97 at OUAL. In addition to the issue of neutron activation, we have also studied the possible production of free hydrogen isotopes within lithium salts as a result of fast neutron bombardment (free hydrogen is highly corrosive). Our detailed numerical simulations have again shown that this should not pose a problem.

Finally, we are currently in the process of evaluating the suitability of several types of targets proposed for use as neutron sources. Once again, our requirements call for a source with a sustained output $\approx 10^{12} / 4 \pi \mathrm{n} / \mathrm{s} / \mathrm{sr}$ in the forward direction, an effective spot size $\approx 1 \mathrm{~mm}$, and an operationally indefinite lifetime. At the present time, we are investigating the possibility of using an accelerator-based DD neutron generator with either a high-pressure $(\approx 1$ atm) windowless deuterium gas target or a sealed gas target with a thin Be window which would rotate at a high angular velocity to distribute the heat loads expected in a full-up imaging facility.

\section{ACKNOWLEDGMENTS}

We would like to thank Professor Roger Finlay and the staff of the Ohio University Accelerator Laboratory in Athens, Ohio for their expert assistance in carrying out experiments crucial to the success of this project.

This work was performed at the University of California, Lawrence Livermore National Laboratory under the auspices of the U.S Department of Energy (contract \# W-7405-Eng-48).

\section{REFERENCES}

[1] A. Gavron, High Energy Neutron Radiography - Observing the LiD, P-23 (Los Alamos National Laboratory) memorandum written on behalf of A. Bridge, C. Morris, M. Murray, S. Seestrom, J. Ullmann, W. Abfalterer, and A. Gavron of LANL and F. Dietrich and T. Wenaus of LLNL (December 7, 1994).

[2] T. Wilcox and E. Lent, COG - Volume 1: User Manual, LLNL Rept. \# M-221-1 (1989); see also R. Buck and E. Lent, "COG: A New, High-Resolution Code for Modeling Radiation Transport," LLNL Energy and Technology Review (June 1993), LLNL Rept. \#. UCRL-52000-93-6.

\section{COLLECTED FIGURES}

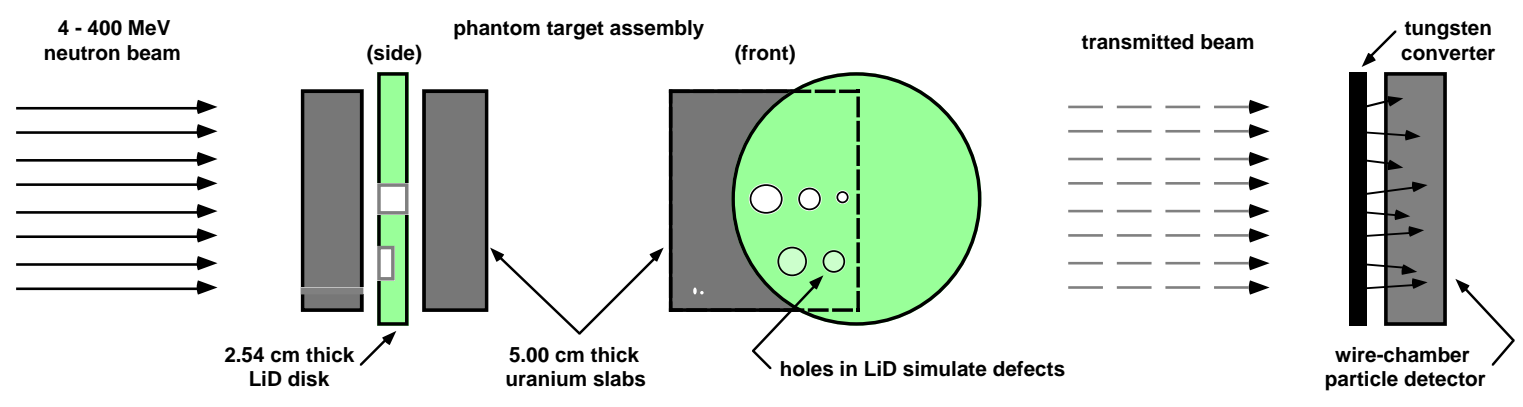

Figure 1: Recent experiments carried out at the LANSCE facility at LANL have demonstrated the potential for using fast neutron radiography for stockpile stewardship. Neutrons with energies of up to $\approx 400 \mathrm{MeV}$ were used to irradiate a uranium and LiD phantom target assembly. Small holes (4-12 mm) drilled all or part of the way through the $\mathrm{LiD}$ were used to simulate defects. A position sensitive multiwire detector (spatial resolution $\approx 1 \mathrm{~mm}$, detection efficiency $\approx 0.3 \%$ ) was used to record the image. 


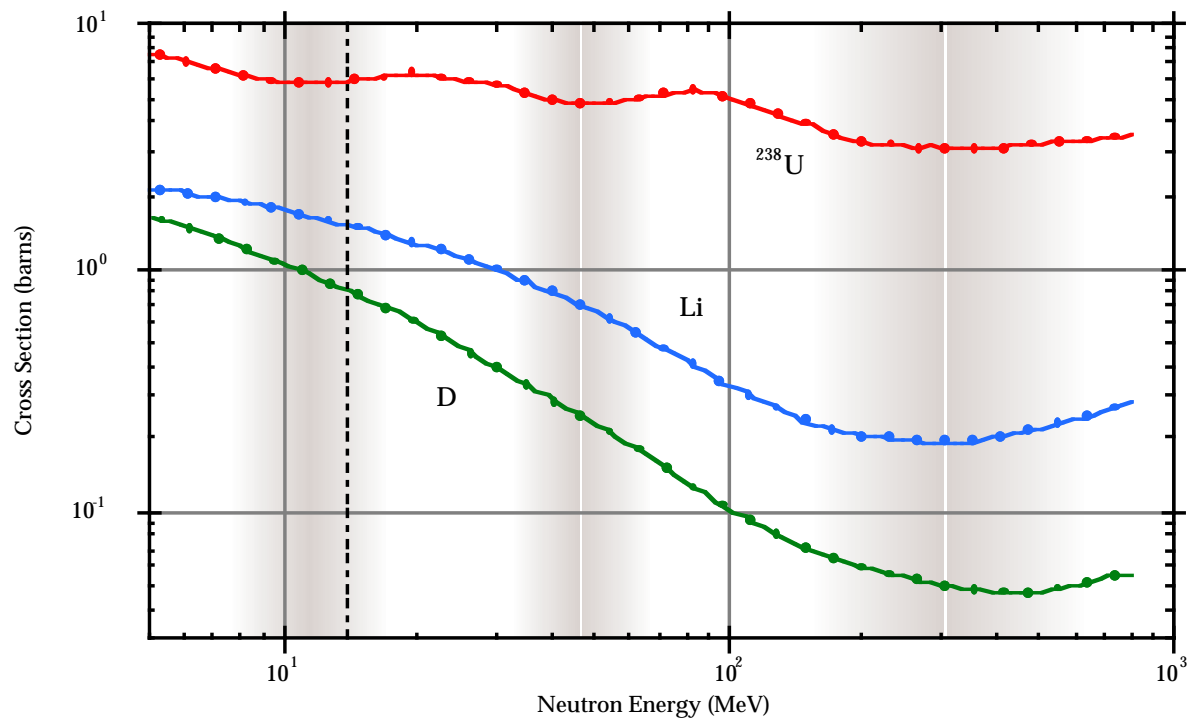

Figure 2: The total neutron interaction cross sections shown here were obtained from A. Gavron (LANL). Dips in the uranium cross section determine energy "windows" suitable for neutron imaging (i.e., regions of relatively high transmission). The higher the lithium and deuterium cross sections are in these regions, the higher the transmission contrast ratio is, thus making it easier to detect defects. The dashed line in the figure marks $14 \mathrm{MeV}$.

LANSCE Experimental Image $(\approx 40-400 \mathrm{MeV})$

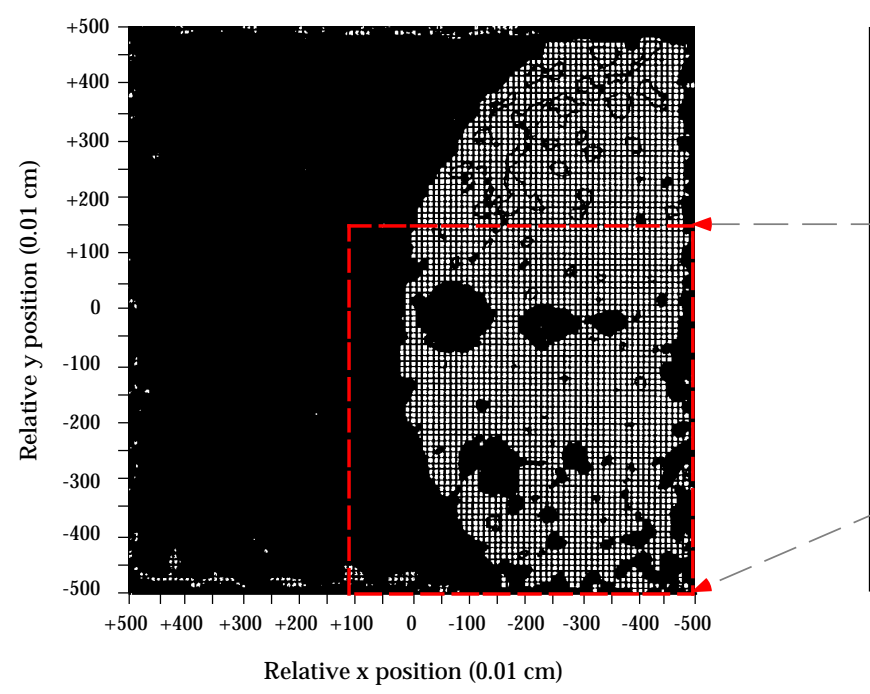

COG Simulation $(14 \mathrm{MeV})$

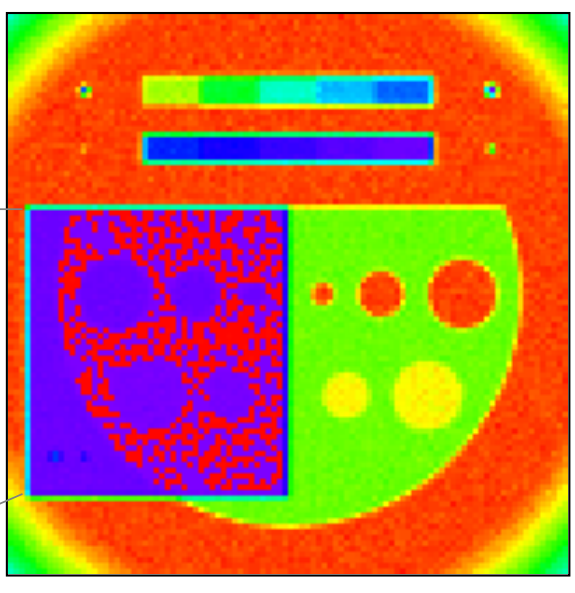

Figure 3: This figure shows the experimental image of the LANSCE phantom target taken using $\approx 40-400 \mathrm{MeV}$ neutrons compared to the COG Monte Carlo simulation of a similar phantom for the case of $14 \mathrm{MeV}$ neutrons. Lineouts taken through corresponding regions in the images show similar contrast ratios in the vicinity of the LiD defects in spite of the large difference in incident neutron energies. 


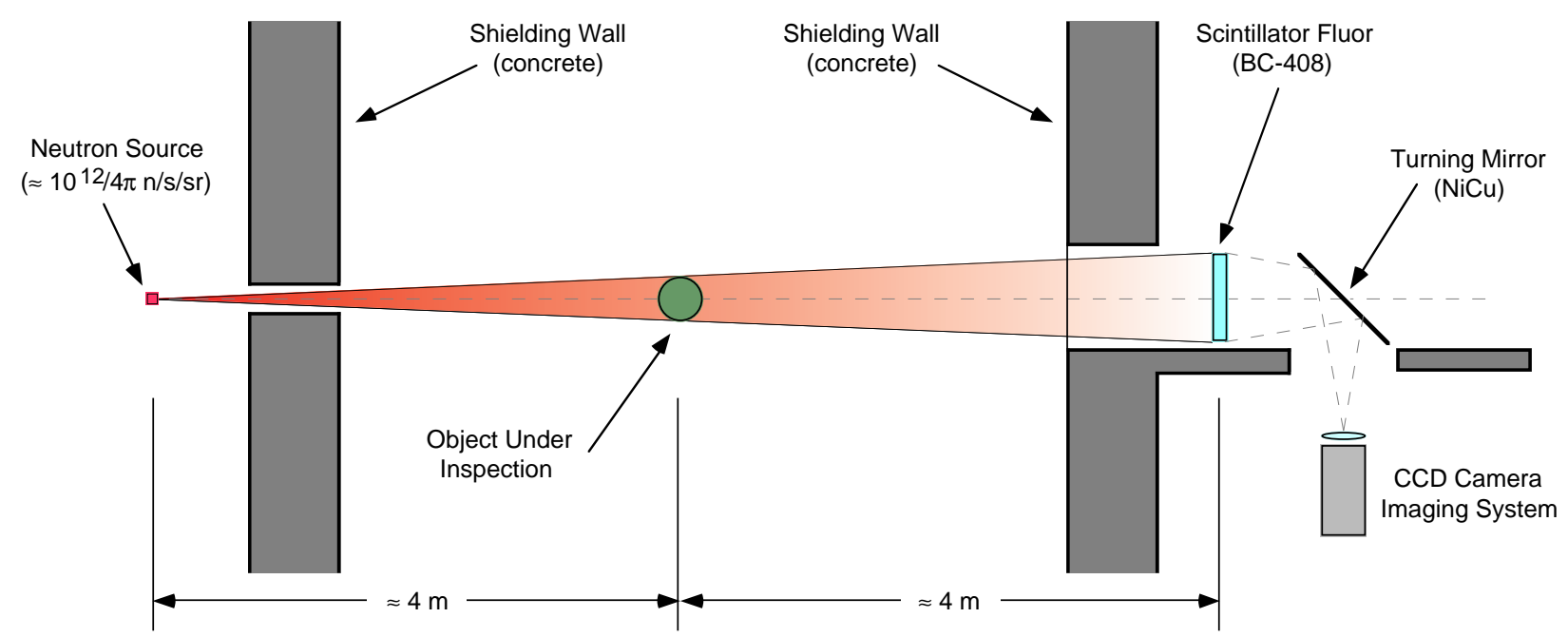

Figure 4: The fast neutron imaging system that we have proposed for use in nuclear stockpile stewardship applications will consist of three primary components: a high-luminosity neutron source operating in the $10-15 \mathrm{MeV}$ energy range (output $\approx 10^{12} / 4 \pi \mathrm{n} / \mathrm{s} / \mathrm{sr}$ in the forward direction, effective spot size $\approx 1 \mathrm{~mm}$ in diameter), a low-mass rotation stage to support and manipulate the object under inspection (allowing for tomographic imaging) and the imaging package itself. Shielding walls will be used as necessary to mitigate scattered radiation backgrounds.

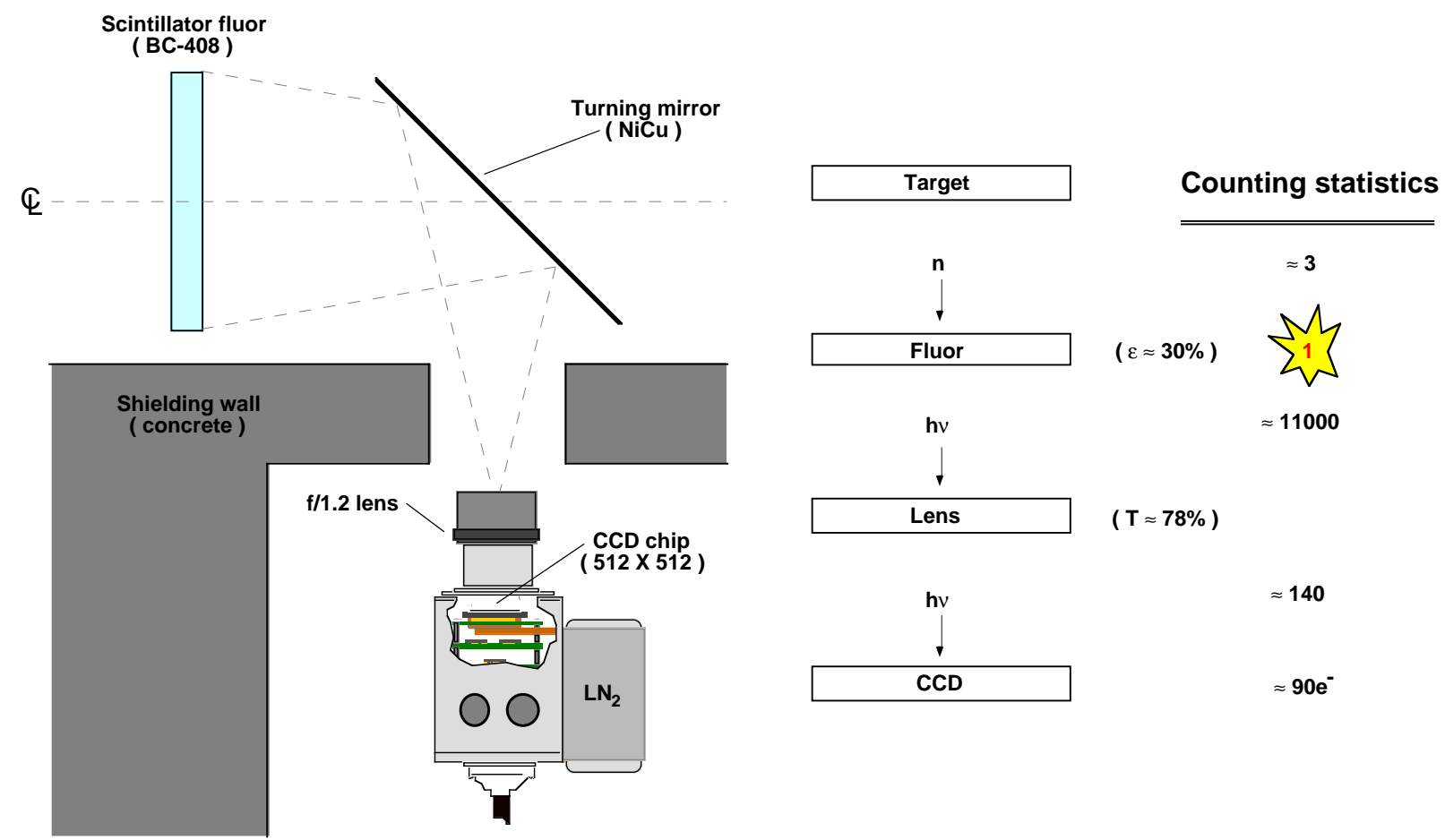

Figure 5: The imaging package will consist of a fast (f/1.2) lens coupled to a back-thinned, high-resolution $(\approx 512$ $X$ 512) CCD imaging chip operating at liquid nitrogen temperatures to minimize thermal electronic noise. A thin metallic turning mirror will be used to reflect light from the scintillator fluor into the camera system which will be located in a shielded enclosure well out of the neutron beam path. 


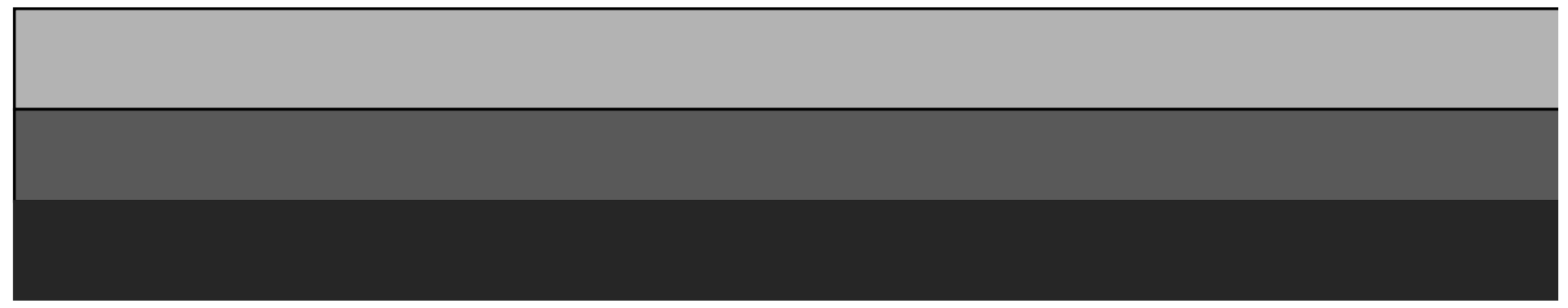

